\title{
Search for nuclearites with the ANTARES detector
}

\section{Bouta, ${ }^{a, *}$ J. Brunner, ${ }^{b}$ A. Moussa, ${ }^{a}$ G. E. Păvălaş ${ }^{c}$ and Y. Tayalati ${ }^{d}$ on behalf of the ANTARES Collaboration}

(a complete list of authors can be found at the end of the proceedings)

${ }^{a}$ University Mohammed First in Oujda, 67600, Oujda, Morocco

${ }^{b}$ Centre de Physique des Particules de Marseille, France

${ }^{c}$ Institute of Space Science, Măgurele, Romania

${ }^{d}$ University Mohammed $V$ in Rabat, Morocco

E-mail: boutamohammed@hotmail.com

ANTARES is a Cherenkov underwater neutrino telescope operating in the Mediterranean. Its construction was completed in 2008. Even though optimised for the search of cosmic neutrinos, this telescope is also sensitive to nuclearites (massive nuggets of strange quark matter [1]) trough the black body radiation emitted along their path [2].

We discuss here the possible detection of non-relativistic down-going nuclearites with the ANTARES telescope and present the results of an analysis using data collected from 2009 till 2017.

$37^{\text {th }}$ International Cosmic Ray Conference (ICRC 2021)

July 12 th - 23rd, 2021

Online - Berlin, Germany

\footnotetext{
${ }^{*}$ Presenter
} 


\section{Introduction}

In 1984 E. Witten proposed a new state of hadronic matter called Strange Quark Matter (SQM), it is made up of roughly equal proportion of up, down, and strange quarks confined in a single hadronic bag that can be small as ordinary nuclei (baryon number $\mathrm{A}=2$ ) or large as a neutron star (baryon number $\mathrm{A} \simeq 2 \times 10^{57}$ ). SQM could be the true ground state for hadronic matter [3]. Macroscopic quark matter lumps surviving from the cosmological quark-hadron phase transition are often referred to as quark nuggets, and if they manage to reach the Earth they are called nuclearites [3-5].

Nuclearites would be expected to have galactic velocities and could be identified by tracks seen in cosmic ray detectors based on the Earth. Nuclearites of galactic velocities are protected by their surrounding electrons against direct interactions with the atoms constituting the traversed medium, and they would likely be neutral but would lose a large amount of energy by elastic and quasi-elastic collisions with atoms and molecules in their path, and so leave distinct tracks in cosmic ray detectors. In the case of a transparent medium, nuclearites signal could be tracked by using their visible light emission as a black-body radiation from an expanding cylindrical thermal shock wave [1].

In this work, we study cosmic nuclearites falling on the Earth with galactic velocities $\left(\beta=10^{-3}\right)$, we consider a mass of $4 \times 10^{13} \mathrm{GeV} / \mathrm{c}^{2}$ as a threshold mass for nuclearites detection at ANTARES level. By using a dedicated Monte Carlo, we simulated both nuclearites and atmospheric muons, which correspond to the main background source. We present here, the results obtained for searching for cosmic nuclearites by using nine years of data.

\section{The ANTARES neutrino telescope}

ANTARES is a Cherenkov based neutrino telescope operating since 2008 in its full configuration. It consists of a three-dimensional array of 885 photomultiplier tubes (PMTs) embedded in optical modules (OMs) and arranged in 12 vertical detection lines deployed at a depth of $2450 \mathrm{~m}$ in the Mediterranean Sea $42 \mathrm{~km}$ offshore in south of Toulon in France. Each line holds 25 floors with three OMs and has about $450 \mathrm{~m}$ length. The distance from floor-to-floor is $14.5 \mathrm{~m}$ and the first floor of each line is located $100 \mathrm{~m}$ from the bottom of the sea while the separation between different lines is of 60 to $75 \mathrm{~m}$. Each line is anchored to the bottom of the sea with the bottom string socket and a dead weight, and is held vertical by a buoy at the top. The detector covers a surface area of $0.1 \mathrm{~km}^{2}$. A full description of the ANTARES detector can be found in [6].

When a photon arrives to the PMT photo-cathode, it can induce an electric signal on the its anode with a probability to occur determined by the PMT quantum efficiency. L0 threshold are hits satisfying the threshold condition (typically set to 0.3 p.e), L1 hits are defined as, either a coincidence of two L0 hits from the same storey within $20 \mathrm{~ns}$, or a single hit with high amplitude exceeding a predefined High-Threshold condition (set to 3 p.e. or 10 p.e. depending on the data taking conditions). 


\section{Energy loss of nuclearites}

The principal energy-loss mechanism for a nuclearite passing through matter is atomic collision. For a massive nuclearite the energy loss rate is [1]:

$$
-\frac{d E}{d x}=\rho \sigma v^{2}
$$

$\rho$ is the density of the traversed medium, $\sigma$ the effective cross-sectional area of nuclearite and $v$ its velocity. The effective area can be obtained by the nuclearite density $\rho_{N}$.

$$
\sigma\left(\mathrm{cm}^{2}\right)=\left\{\begin{array}{lll}
\pi \cdot 10^{-16} & \text { if } & M_{n}<8.4 \cdot 10^{14} \mathrm{GeV} / \mathrm{c}^{2} \\
\pi \cdot\left(\frac{3 M_{n}}{4 \pi \rho_{N}}\right) & \text { if } & M_{n} \geq 8.4 \cdot 10^{14} \mathrm{GeV} / \mathrm{c}^{2}
\end{array}\right.
$$

where $M_{N}$ is the nuclearite mass and $\rho_{N}=3.5 \times 10^{14} \mathrm{~g} / \mathrm{cm}^{3}$ is the its density.

We assume that nuclearites enter the atmosphere with a typical velocity of $\beta_{0}=\frac{v_{0}}{c}=10^{-3}$, before it reaches the ANTARES volume it should propagate throw the atmosphere and interact with its constituent. At the sea level nuclearites should have a velocity of $\beta<\beta_{0}$, and it is propagated to ANTARES volume throw water where the density was taken $\rho_{w}=1 \mathrm{~g} \mathrm{~cm}^{-3}$. For nuclearite of mass $M_{n}$ penetrating at depth of $L$ in medium of density $\rho$, its velocity changes as [1]:

$$
\beta(L)=\beta_{0} \cdot \exp \left(\frac{\sigma}{M_{n}} \int_{0}^{L} \rho(x) d x\right)
$$

We use the parametrization of the standard atmosphere given in equation 4 [7], to propagate nuclearites over the atmosphere :

$$
\begin{aligned}
\rho(x) & =a \cdot \exp \left(-\frac{x}{b}\right) \\
& =a \cdot \exp \left(-\frac{H-L}{b}\right)
\end{aligned}
$$

Where $a=1.2 \times 10^{-3} \mathrm{~g} \mathrm{~cm}^{-3}$ and $b \simeq 8.57 \times 10^{5} \mathrm{~cm}$ and $H \simeq 50 \mathrm{~km}$ is the height of the atmosphere.

In the case of a transparent medium, nuclearites signal could be tracked by using their visible light emission as a black-body radiation from an expanding cylindrical thermal shock wave [5], the fraction of energy transformed in light called luminous efficiency $\eta$ it was estimated to be $\eta \simeq 3 \times 10^{-5}$ in the case of water; thus, the number of visible photons radiated per unit of path length is estimated as [8]:

$$
\frac{d N_{\gamma}}{d x}=\frac{\eta}{\pi(e V)} \cdot \frac{d E}{d x}
$$




\section{Search for nuclearites in the ANTARES detector}

To avoid any biased results, a fraction of experimental data is used for comparing real data and the Monte Carlo simulation. Due to the environmental optical background wich affecting our analysis, a selection conditions are applied for the runs samples in order to select a clean data set. We selected only runs which are not significantly affected by bioluminescence or other known hardware problems.

The Monte Carlo simulation of nuclearite in ANTARES is performed using a dedicated software, a fortran program, Antsim, generate these particles with a random zenith and azimuth angles on an abstract hemisphere of a radius of $548 \mathrm{~m}$ surrounding the detector. The velocity and the energy loss are evaluated using formulas described in section 3 , each particle proceeds with a time step of $2 \mathrm{~ns}$ in its direction and the light is generated for every iteration using equation 5 . The detector response was simulated using the standard software tools [9]. For our study, atmospheric muons will constitute the main background events source. These particles are simulated using the MUPAGE package.

We aim to isolate the nuclearites signal form any other particles which could be detected by ANTARES. Taking into account the physical properties of these particles and the detection conditions, a set of cuts and trigger selection are applied to each event. We select only events triggered by 3D and/or T3 triggers, and we apply a quality cut where only events with at least 300 L0 hits are accepted. This cut allows to reduce the optical noise effect presented in the real data, it allows also to remove the atmospheric muons events of high amplitude and low number of floors causing a high luminous weight of the events in the detector.

In order to separate our signal from the background, we use a discrimination variables, the first one is $\log _{10}$ (nhits 3 )/nfloor, it is a combination of the nhits 3 variable which is the number of hits with charge of at least three photo-electrons (p.e.) for a given event, while the nfloor variable stands for the number of floors which recorded at least one hit. Figure 1 shows $\log _{10}$ (nhits3)/nfloor distribution for differents samples.

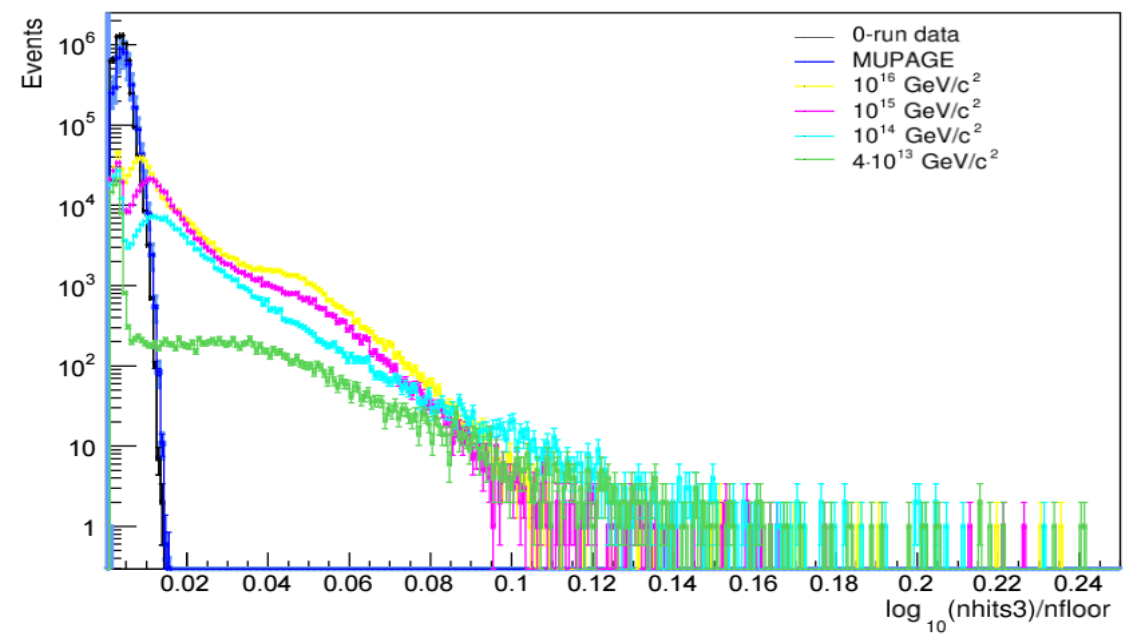

Figure 1: $\log _{10}$ (nhits3)/nfloor distribution after applying a cut of $\mathrm{L} 0 \geq 300$. Black for a $10 \%$ subset of the real data sample, blue for atmospheric muons and with other colors for each nuclearites mass. 
Nuclearites, as they are slow heavy particles, are expected to have a high transit time in the detector, dt. If we combine $\log _{10}$ (nhits3)/nfloor variable with the dt variable which refers to the time spent by an event in the detector, we will be able to isolate our signal. The $2 \mathrm{D}$ plot for these two variable is presented in figure 2 .

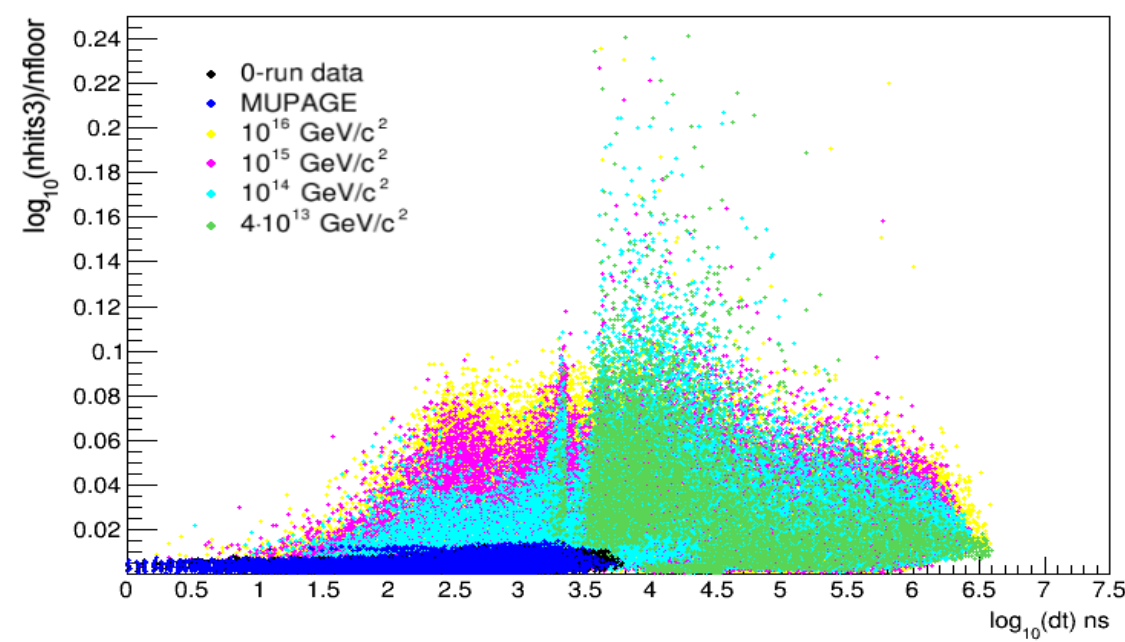

Figure 2: A 2D plot representing the $\log _{10}$ (nhits3)/nfloor versus dt. In black for real data, blue for atmospheric muons and for each nuclearites mass with other colors. As it can be seen nuclearites are well isolated.

\section{Optimization}

Once we define our discrimination variables, we should calculate the best cuts on these two variables in order to isolate the nuclearites from other particles constituting a background. This means to find a couple of values on both parametres in such a way that maximize the signal and minimize as much as possible the background events. A dedicated algorithm wich scan a $2 \mathrm{D}$ histogram of $\log _{10}$ (nhits3)/nfloor versus dt with small steps and calculate the best values of the optimization as shown in figure 3.

In order to compensate for the lack of statistics in both $\log _{10}$ (nhits3)/nfloor and dt distribution, we made an extrapolation in the signal region for the real data as it is affected by bio-activity and ${ }^{40} \mathrm{~K}$ decay in that region. The extrapolation is made by fitting the two distributions with a Landau type distribution. This extrapolation was taken ito account for the minimisation of the cuts on $\log _{10}$ (nhits3)/nfloor and dt variables.

To calculate the best sensitivity of ANTARES to nuclearites without any biased results, we use the Model Rejection Factor (MRF). By using a dedicated algorithm, we look for the best cuts on $d t$ and $\log _{10}$ (nhits3)/nfloor that minimizing the MRF for each nuclearites mass.

The sensitivity at $90 \%$ of C.L. noted $S_{90}$ is computed using the Feldman-Cousins formula assuming events with a Poissonian distribution.

$$
S_{90}\left(c m^{-2} \cdot s r^{-1} \cdot s^{-1}\right)=\frac{\bar{\mu}_{90}\left(n_{b}\right)}{S_{e f f}\left(c m^{2} \cdot s r\right) \times T(s)}
$$


Where T is the duration of data taking corresponding to 2009-2017 period in seconds, $\bar{\mu}_{90}$ and $S_{\text {eff }}$ are defined as follows:

$$
\begin{gathered}
\bar{\mu}_{90}=\sum_{n_{o b s}=1}^{\infty} \mu_{90}\left(n_{o b s}, n_{b}\right) \cdot \frac{n_{b}^{n_{o b s}}}{n_{o b s} !} \cdot e^{-n_{b}} \\
S_{\text {eff }}=\frac{n_{N u c}}{\Phi_{N u c}}
\end{gathered}
$$

$n_{N u c}$ represent the number of nuclearites remaining after applying the optimized cuts, and $\Phi_{N u c}$ represent the flux of generated Nuclearites. The Model Rejection Factor consists of varying the cuts with small steps until the minimum of MRF is reached, which corresponds to the values that gives the best sensitivity.
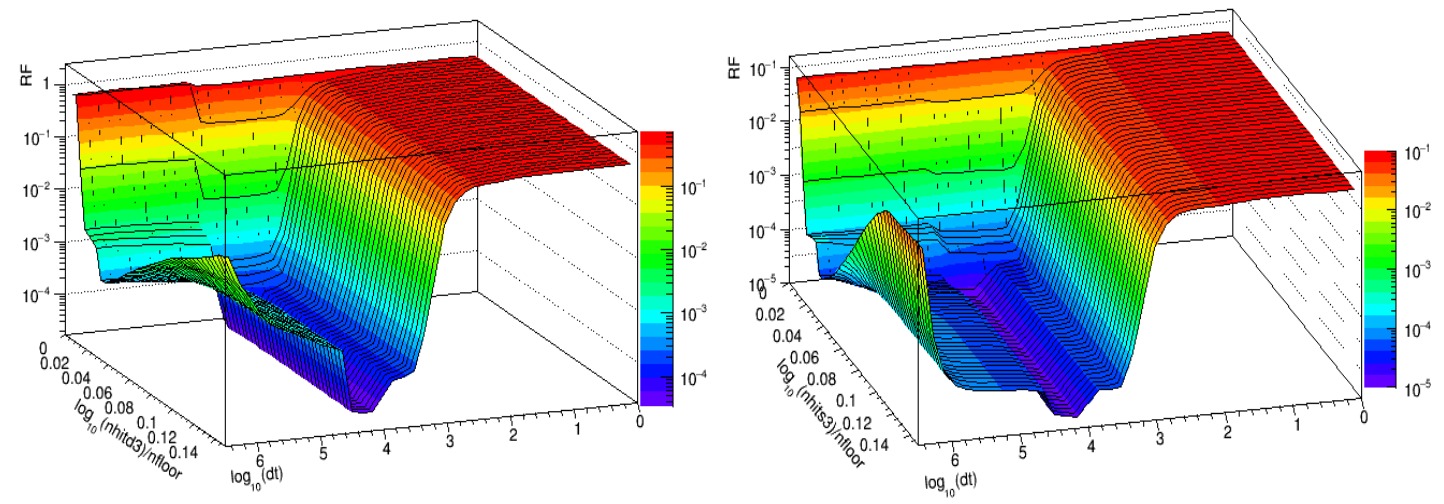

Figure 3: Examples of rejection factors. On the left, the rejection factor for a given set of cuts on $\log _{10}$ (nhits 3 )/nfloor and $\mathrm{dt}$ for $4 \times 10^{13} \mathrm{GeV} / \mathrm{c}^{2}$. on the right, the same plot for the higher mass of nuclearite (i.e $10^{16} \mathrm{GeV} / \mathrm{c}^{2}$ ). The best cuts are those where the MRF is minimal.

\section{Sensitivity results}

The sensitivity obtained by ANTARES for the period 2009-2017 Corresponding to 839 days of livetime of the detector is shown in Figure 4, in this figure we also drew the upper limits on the flux obtained by other experiments such as SLIM and MACRO, and the upper limits for 2009 period carried out in the previous nuclearites analysis with the ANTARES telescope [10-12]. As it can be seen, ANTARES is sensitive to slow moving heavy particles even in the absence of a dedicated trigger for such particules. For masses higher than $10^{16} \mathrm{GeV} / \mathrm{c}^{2}$, nuclearites events must be more energetic and they would emit more light. Therefore, the limit of the last test point can be taken as a conservative limit also for larger nuclearites masses.

If no candidate found in the forthcoming analyses, the new flux limit will improve the MACRO and SLIM upper limits. The following table summarizes the values of differents parameters of the analysis. 


\begin{tabular}{l|cccc} 
& $10^{16} \mathrm{GeV} / \mathrm{c}^{2}$ & $10^{15} \mathrm{GeV} / \mathrm{c}^{2}$ & $10^{14} \mathrm{GeV} / \mathrm{c}^{2}$ & $4 \cdot 10^{13} \mathrm{GeV} / \mathrm{c}^{2}$ \\
\hline Best cut on $\log _{10}(\mathrm{dt})$ & $\geq 4.125$ & $\geq 4.125$ & $\geq 4.125$ & $\geq 4.125$ \\
Best cut on $\log _{10}(\mathrm{nhits} 3) / \mathrm{nfloor}$ & $\geq 0.029$ & $\geq 0.029$ & $\geq 0.029$ & $\geq 0.029$ \\
Remaining nuclearites & 213711 & 172160 & 119743 & 71964 \\
Remaining background & 0 & 0 & 0 & 0 \\
Minimal RF & $1.14 \times 10^{-05}$ & $1.41 \times 10^{-05}$ & $2.03 \times 10^{-05}$ & $3.39 \times 10^{-05}$ \\
Sensitivity $\left(\mathrm{cm}^{-2} \cdot \mathrm{sr}^{-1} \cdot \mathrm{s}^{-1}\right)$ & $4.61 \times 10^{-18}$ & $5.72 \times 10^{-18}$ & $8.23 \times 10^{-18}$ & $1.37 \times 10^{-17}$ \\
\hline
\end{tabular}

Table 1: Values of the optimized parameters and the results of the minimal RF and sensitivities for the different nuclearites masses for 2009-2017 period.

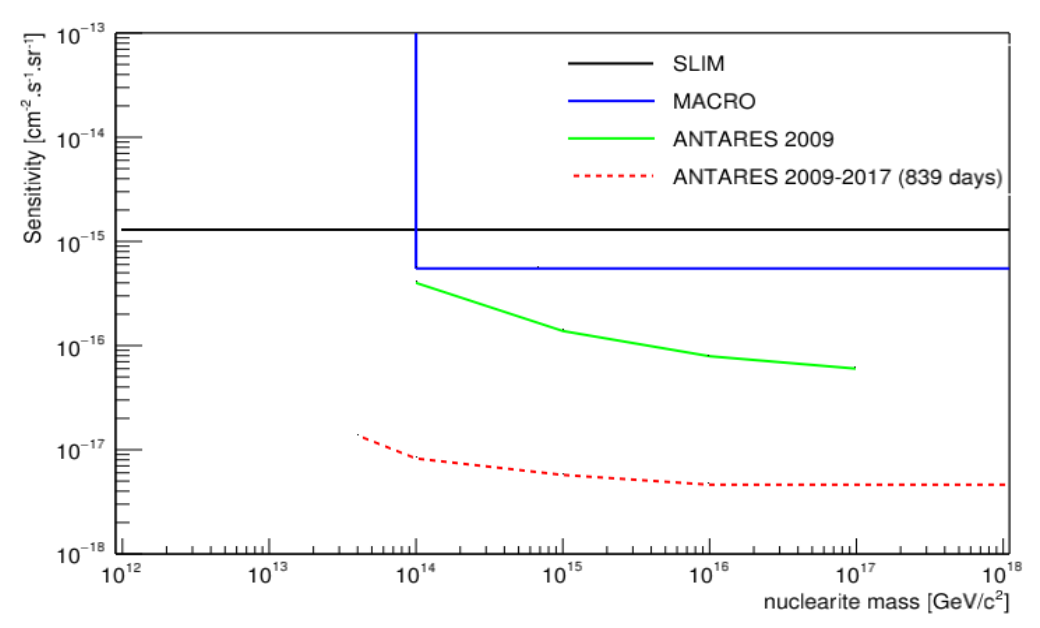

Figure 4: Sensitivity of ANTARES to down-going flux of nuclearites for 839 days of data taking.

\section{Conclusion}

This work presents the analysis performed for search for down-going heavy and slow moving nuclearites assuming a velocity at the entrance of the atmosphere of $\beta_{0}=10^{-3}$. Nuclearites heavier than $4 \cdot 10^{13} \mathrm{GeV} / \mathrm{c}^{2}$ would be able to reach the ANATRES detector depth with enough energies to generate a sufficient visible photon and to be detected. Our study is based on two discrimination variables that reflect perfectly the nuclearite behaviour in the telescope, a run-by-run version 4 Monte Carlo simulations were used where atmospheric muons and optical background such as bioluminescence and ${ }^{40} \mathrm{~K}$ decay present a background for the analysis.

A burned sample (runs ending with 0 , corresponding to $10 \%$ of the data) of real data is used for the comparison data-MC in the period of 2009-2017. The usage of the model of rejection factor allows us to calculate the optimized cuts on $d t$ and $\log _{10}$ (nhits3)/nfloor takin into account the extrapolated events which make the cuts safer, and so, calculate the best sensitivity of ANTARES to a given mass of nuclearites for the period in consideration.

The result of the sensitivity obtained is competitive to the limits on the flux reported by other experiments such as MACRO and SLIM. It also improves the results already obtained by ANTARES for 2009 period. 


\section{References}

[1] A. De Rújula \& S. L. Glashow, "Nuclearites - a novel form of cosmic radiation". Nature 312 (1984) 734.

[2] V. Popa, "Very large volume neutrino telescopes as magnetic monopole and nuclearite detectors". Nucl. Instr. \& Meths. in Phys. Res. A 567 (2006) 480-482.

[3] Edward Witten, "Cosmic separation of phases". Phys.Rev.D 30 (1984) 272-285.

[4] F. Weber, "Strange Quark Matter and Compact Stars". Prog.Part.Nucl.Phys.54:193-288,2005.

[5] Edward Farhi and R. L. Jaff, "Strange matter". Phys. Rev. D 30, 2379.

[6] M. Ageron et al. [ANTARES], "ANTARES: the first undersea neutrino telescope". Nucl. Instr. \& Meths. A 656 (2011), 11-38.

[7] T. Shibata, Prog.Theor.Phys. 57 (1977) 882

[8] G. E. Păvălaş, "Search for nuclearites with the ANTARES detector". arXiv:1010.2071 [astroph.HE].

[9] A. Albert et al., "Monte Carlo simulations in the ANTARES underwater neutrino telescope". JCAP01 (2021) 064.

[10] M. Ambrosio et al., "Nuclearite search with the MACRO detector at Gran Sasso". Eur. Phys. J. C 13 (2000) 453.

[11] G. E. Păvălaş (ANTARES Collaboration), PoS(ICRC2015)1060.

[12] Z. Sahnoun et. al. "Search for strange quark matter and Q-balls with the SLIM experiment". Radiat.Meas. 44 (2009) 894-897. 


\section{Full Authors List: ANTARES Collaboration}

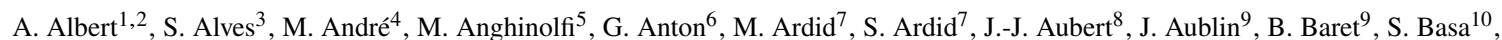
B. Belhorma ${ }^{11}$, M. Bendahman ${ }^{9}, 12$, V. Bertin ${ }^{8}$, S. Biagi ${ }^{13}$, M. Bissinger ${ }^{6}$, J. Boumaaza ${ }^{12}$, M. Bouta ${ }^{14}$, M.C. Bouwhuis ${ }^{15}$, H. Brânzaş ${ }^{16}$, R. Bruijn ${ }^{15,17}$, J. Brunner ${ }^{8}$, J. Busto ${ }^{8}$, B. Caiffi ${ }^{5}$, A. Capone ${ }^{18,19}$, L. Caramete ${ }^{16}$, J. Carr ${ }^{8}$, V. Carretero ${ }^{3}$, S. Celli ${ }^{18,19}$, M. Chabab ${ }^{20}$, T. N. Chau ${ }^{9}$, R. Cherkaoui El Moursli ${ }^{12}$, T. Chiarusi ${ }^{21}$, M. Circella ${ }^{22}$, A. Coleiro ${ }^{9}$, M. Colomer-Molla ${ }^{9,3}$, R. Coniglione ${ }^{13}$, P. Coyle ${ }^{8}$, A. Creusot $^{9}$, A. F. Díaz ${ }^{23}$, G. de Wasseige ${ }^{9}$, A. Deschamps ${ }^{24}$, C. Distefano ${ }^{13}$, I. Di Palma ${ }^{18,19}$, A. Domi ${ }^{15,17}$, C. Donzaud ${ }^{9,25}$, D. Dornic ${ }^{8}$, D. Drouhin ${ }^{1,2}$, T. Eberl ${ }^{6}$, T. van $E^{2}$ eden $^{15}$, D. van Eijk ${ }^{15}$, N. El Khayati ${ }^{12}$, A. Enzenhöfer ${ }^{8}$, P. Fermani ${ }^{18,19}$, G. Ferrara $^{13}$, F. Filippini ${ }^{21,26}$, L.A. Fusco ${ }^{8}$, Y. Gatelet ${ }^{9}$, P. Gay ${ }^{27,9}$, H. Glotin ${ }^{28}$, R. Gozzini ${ }^{3}$, R. Gracia Ruiz ${ }^{15}$, K. Graf ${ }^{6}$, C. Guidi ${ }^{5,29}$, S. Hallmann ${ }^{6}$, H. van Haren ${ }^{30}$, A.J. Heijboer ${ }^{15}$, Y. Hello ${ }^{24}$, J.J. Hernández-Rey ${ }^{3}$, J. Höß1 ${ }^{6}$, J. Hofestädt ${ }^{6}$, F. Huang ${ }^{8}$, G. Illuminati ${ }^{9}, 21,26$, C.W James ${ }^{31}$, B. Jisse-Jung ${ }^{15}$, M. de Jong ${ }^{15,32}$, P. de Jong ${ }^{15}$, M. Kadler ${ }^{33}$, O. Kalekin ${ }^{6}$, U. Katz ${ }^{6}$, N.R. Khan-Chowdhury ${ }^{3}$, A. Kouchner ${ }^{9}$, I. Kreykenbohm ${ }^{34}$, V. Kulikovskiy ${ }^{5,36}$, R. Lahmann ${ }^{6}$, R. Le Breton ${ }^{9}$, D. Lefèvre ${ }^{35}$, E. Leonora ${ }^{36}$, G. Levi ${ }^{21,26}$, M. Lincetto ${ }^{8}$, D. Lopez-Coto ${ }^{37}$, S. Loucatos ${ }^{38,9}$, L. Maderer ${ }^{9}$, J. Manczak ${ }^{3}$, M. Marcelin $^{10}$, A. Margiotta ${ }^{21,26}$, A. Marinelli ${ }^{39}$, J.A. Martínez-Mora ${ }^{7}$, K. Melis ${ }^{15,17}$, P. Migliozzi ${ }^{39}$, A. Moussa ${ }^{14}$, R. Muller ${ }^{15}$, L. Nauta ${ }^{15}$, S. Navas ${ }^{37}$, E. Nezri ${ }^{10}$, B. O' Fearraigh $^{15}$, A. Păun ${ }^{16}$, G.E. Păvălaş ${ }^{16}$, C. Pellegrino ${ }^{21,40,41}$, M. Perrin-Terrin ${ }^{8}$, V. Pestel ${ }^{15}$, P. Piattelli ${ }^{13}$, C. Pieterse ${ }^{3}$, C. Poirè ${ }^{7}$, V. Popa ${ }^{16}$, T. Pradier ${ }^{1}$, N. Randazzo ${ }^{36}$, S. Reck $^{6}$, G. Riccobene ${ }^{13}$, A. Romanov ${ }^{5,29}$, A. Sánchez-Losa ${ }^{3,22}$, F. Salesa Greus ${ }^{3}$, D. F. E. Samtleben ${ }^{15,32}$, M. Sanguineti ${ }^{5,29}$, P. Sapienza ${ }^{13}$, J. Schnabel ${ }^{6}$, J. Schumann ${ }^{6}$, F. Schüssler ${ }^{38}$, M. Spurio ${ }^{21,26}$, Th. Stolarczyk ${ }^{38}$, M. Taiuti ${ }^{5,29}$, Y. Tayalati ${ }^{12}$, S.J. Tingay ${ }^{31}$, B. Vallage ${ }^{38,9}$, V. Van Elewyck ${ }^{9,41}$, F. Versari ${ }^{21,26,9}$, S. Viola ${ }^{13}$, D. Vivolo ${ }^{39,43}$, J. Wilms ${ }^{34}$, S. Zavatarelli ${ }^{5}$, A. Zegarelli ${ }^{18,19}$, J.D. Zornoza ${ }^{3}$, and J. Zúñiga ${ }^{3}$

${ }^{1}$ Université de Strasbourg, CNRS, IPHC UMR 7178, F-67000 Strasbourg, France. ${ }^{2}$ Université de Haute Alsace, F-68100 Mulhouse, France. ${ }^{3}$ IFIC - Instituto de Física Corpuscular (CSIC - Universitat de València) c/ Catedrático José Beltrán, 2 E-46980 Paterna, Valencia, Spain. ${ }^{4}$ Technical University of Catalonia, Laboratory of Applied Bioacoustics, Rambla Exposició, 08800 Vilanova i la Geltrú, Barcelona, Spain. ${ }^{5}$ INFN - Sezione di Genova, Via Dodecaneso 33, 16146 Genova, Italy. ${ }^{6}$ Friedrich-Alexander-Universität Erlangen-Nürnberg, Erlangen Centre for Astroparticle Physics, Erwin-Rommel-Str. 1, 91058 Erlangen, Germany. ${ }^{7}$ Institut d'Investigació per a la Gestió Integrada de les Zones Costaneres (IGIC) - Universitat Politècnica de València. C/ Paranimf 1, 46730 Gandia, Spain. ${ }^{8}$ Aix Marseille Univ, CNRS/IN2P3, CPPM, Marseille, France. ${ }^{9}$ Université de Paris, CNRS, Astroparticule et Cosmologie, F-75013 Paris, France. ${ }^{10}$ Aix Marseille Univ, CNRS, CNES, LAM, Marseille, France. ${ }^{11}$ National Center for Energy Sciences and Nuclear Techniques, B.P.1382, R. P.10001 Rabat, Morocco. ${ }^{12}$ University Mohammed V in Rabat, Faculty of Sciences, 4 av. Ibn Battouta, B.P. 1014, R.P. 10000 Rabat, Morocco. ${ }^{13}$ INFN - Laboratori Nazionali del Sud (LNS), Via S. Sofia 62, 95123 Catania, Italy. ${ }^{14}$ University Mohammed I, Laboratory of Physics of Matter and Radiations, B.P.717, Oujda 6000, Morocco. ${ }^{15}$ Nikhef, Science Park, Amsterdam, The Netherlands. ${ }^{16}$ Institute of Space Science, RO-077125 Bucharest, Măgurele, Romania. ${ }^{17}$ Universiteit van Amsterdam, Instituut voor Hoge-Energie Fysica, Science Park 105, 1098 XG Amsterdam, The Netherlands. ${ }^{18}$ INFN - Sezione di Roma, P.le Aldo Moro 2, 00185 Roma, Italy. ${ }^{19}$ Dipartimento di Fisica dell’Università La Sapienza, P.le Aldo Moro 2, 00185 Roma, Italy. ${ }^{20}$ LPHEA, Faculty of Science - Semlali, Cadi Ayyad University, P.O.B. 2390, Marrakech, Morocco. ${ }^{21}$ INFN - Sezione di Bologna, Viale Berti-Pichat 6/2, 40127 Bologna, Italy. ${ }^{22}$ INFN - Sezione di Bari, Via E. Orabona 4, 70126 Bari, Italy. ${ }^{23}$ Department of Computer Architecture and Technology/CITIC, University of Granada, 18071 Granada, Spain. ${ }^{24}$ Géoazur, UCA, CNRS, IRD, Observatoire de la Côte d'Azur, Sophia Antipolis, France. ${ }^{25}$ Université Paris-Sud, 91405 Orsay Cedex, France. ${ }^{26}$ Dipartimento di Fisica e Astronomia dell'Università, Viale Berti Pichat 6/2, 40127 Bologna, Italy. ${ }^{27}$ Laboratoire de Physique Corpusculaire, Clermont Université, Université Blaise Pascal, CNRS/IN2P3, BP 10448, F-63000 Clermont-Ferrand, France. ${ }^{28}$ LIS, UMR Université de Toulon, Aix Marseille Université, CNRS, 83041 Toulon, France. ${ }^{29}$ Dipartimento di Fisica dell’Università, Via Dodecaneso 33, 16146 Genova, Italy. ${ }^{30}$ Royal Netherlands Institute for Sea Research (NIOZ), Landsdiep 4, 1797 SZ 't Horntje (Texel), the Netherlands. ${ }^{31}$ International Centre for Radio Astronomy Research - Curtin University, Bentley, WA 6102, Australia. ${ }^{32}$ Huygens-Kamerlingh Onnes Laboratorium, Universiteit Leiden, The Netherlands. ${ }^{33}$ Institut für Theoretische Physik und Astrophysik, Universität Würzburg, Emil-Fischer Str. 31, 97074 Würzburg, Germany. ${ }^{34}$ Dr. Remeis-Sternwarte and ECAP, Friedrich-Alexander-Universität Erlangen-Nürnberg, Sternwartstr. 7, 96049 Bamberg, Germany. ${ }^{35}$ Mediterranean Institute of Oceanography (MIO), Aix-Marseille University, 13288, Marseille, Cedex 9, France; Université du Sud Toulon-Var, CNRS-INSU/IRD UM 110, 83957, La Garde Cedex, France. ${ }^{36}$ INFN - Sezione di Catania, Via S. Sofia 64, 95123 Catania, Italy. ${ }^{37}$ Dpto. de Física Teórica y del Cosmos \& C.A.F.P.E., University of Granada, 18071 Granada, Spain. ${ }^{38}$ IRFU, CEA, Université Paris-Saclay, F-91191 Gif-sur-Yvette, France. ${ }^{39}$ INFN - Sezione di Napoli, Via Cintia 80126 Napoli, Italy. ${ }^{40}$ Museo Storico della Fisica e Centro Studi e Ricerche Enrico Fermi, Piazza del Viminale 1, 00184, Roma. ${ }^{41}$ INFN - CNAF, Viale C. Berti Pichat 6/2, 40127, Bologna. ${ }^{42}$ Institut Universitaire de France, 75005 Paris, France. ${ }^{43}$ Dipartimento di Fisica dell'Università Federico II di Napoli, Via Cintia 80126, Napoli, Italy. 